\title{
Utilização de Haar e Spline Wavelets para analisar sinais cardíacos de fetos
}

\author{
Marcelo C. Rossato* Alice Kozakevicius \\ Universidade Federal de Santa Maria - Departamento de Matemática \\ 97015-900, Santa Maria, RS \\ E-mail: marcelocrossato_1@ hotmail.com, alice.kozakevicius@gmail.com.
}

\section{RESUMO}

Este trabalho tem como objetivo analisar eletrocardiogramas e separar os sinais cardíacos de fetos do sinal cardíaco materno e do ruído, utilizando como ferramenta as transformadas de Haar e Spline Wavelets, verificando os diferentes resultados encontrados com essas duas famílias de Wavelets.

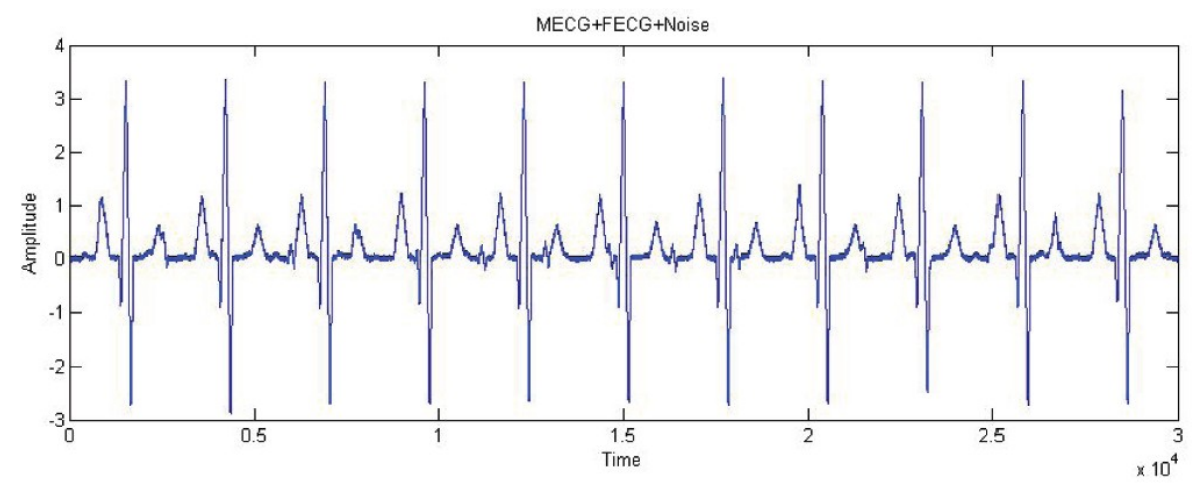

Figura 1: Eletrocardiograma contendo sinal da mãe, sinal do feto e ruído[4]

Como é possível perceber no ECG da Figura 1 acima, enquanto os picos do sinal da mãe são facilmente encontrados, o sinal cardíaco do feto é de difícil observação e pode ser facilmente confundido com o ruído. Desse modo, é essencial encontrar um método para se poder analisar adequadamente esses sinais, pois isso facilitaria muito na identificação de problemas relacionados ao desenvolvimento do feto.

Além disso, é importante ressaltar que as características dos sinais do feto sofrem grandes alterações durante o período de gestação, fazendo com que não seja possível identificar uma única forma de onda[5]. Nesta pesquisa, busca-se encontrar padrões desses sinais com o auxílio das transformadas de Haar e Spline Wavelets[8,9].

Este trabalho dá continuidade ao estudo apresentado em [1,2], no entanto agora o enfoque será na construção de spline wavelets para a análise de sinais cardíacos. Uma outra questão a ser abordada é quanto à influência da intensidade do ruído no processo de filtragem e obtenção de uma aproximação satisfatória para o sinal do feto.

Wavelets são bases de um espaço vetorial e podem ser utilizadas para analisar a decomposição de funções. Essas funções podem ser representados por um valor médio, associado a detalhes que variam dos níveis mais finos aos mais grosseiros.

As Wavelets ortonormais, como as Haar Wavelets, são decompostas a partir de um método denominado análise multiresolução. O ponto de partida dessa análise é de que temos um conjunto de espaços vetoriais onde cada um está contido no seguinte, ou seja: $V^{0} \subset V^{1} \subset V^{2} \subset \ldots$

\footnotetext{
*bolsista de Iniciação Científica CAPES
} 
À medida que $\mathrm{j}$ aumenta, a resolução de $V^{j}$ aumenta. As funções de escala $\phi^{j}$ formam a base mais comum dos espaços $V^{j}$. Sendo $W^{j}$ o complemento ortogonal de $V^{j}$ em relação a $V^{j+1}$, são denominadas wavelets as funções $\psi^{j}$ que formam uma base para $W^{j}$.

É possível escrever as funções de escala e as funções wavelets em duas matrizes de uma linha única com $m^{j}$ e $n^{j}$ colunas, respectivamente, onde $m^{j}$ é a resolução de $V^{j}$ e $n^{j}$ é a resolução de $W^{j}$ :

$\Phi^{j}(x)=\left[\phi_{0}^{j}(x) \ldots \phi_{m^{j}-1}^{j}(x)\right]$ e $\Psi^{j}(x)=\left[\psi_{0}^{j}(x) \ldots \psi_{n^{j}-1}^{j}(x)\right]$

A partir dessas matrizes, podemos encontrar uma matriz $P^{j}$ que seja $m^{j} \times m^{j-1}$ e outra matriz $Q^{j}$ que seja $m^{j} \times n^{j-1}$, satisfazendo a seguinte condição: $\left[\Phi^{j-1}(x) \mid \Psi^{j-1}(x)\right]=\Phi^{j}(x)\left[P^{j} \mid Q^{j}\right]$.

Essas informações são úteis para mostrar que as Wavelets podem ser representadas na forma de uma matriz, mas a parte mais importante é o banco de filtragem:

Dado um conjunto com $m^{j}$ coeficientes que pode ser representado numa matriz coluna $C^{j}=$ $\left[C_{0}^{j} \ldots c_{m^{j}-1}^{j}\right]$, é possível criar um conjunto $C^{j-1}$ com uma resolução menor e com $m^{j-1}$ coeficientes a partir da equação $C^{j-1}=A^{j} C^{j}$, onde $A^{j}$ é uma matriz $m^{j-1} \times m^{j}$.

Esse processo, entretanto, resulta em uma perda de dados, a qual pode ser capturada em uma matriz $D^{j-1}$ a partir da equação $D^{j-1}=B^{j} C^{j}$, onde $B^{j}$ é uma matriz $n^{j-1} \times m^{j}$.

O banco de filtragem é obtido ao repetir essa decomposição recursivamente até obter um conjunto de dados $C^{0}, D^{0}, D^{1}, \ldots, D^{j-1}$, que é denominado transformada Wavelet. Após isso, é possível reconstruir o conjunto $C^{j}$ a partir de $C^{j-1}$ e $D^{j-1}$ segundo a equação $C^{j}=P^{j} C^{j-1}+Q^{j} D^{j-1}$.

Para a escolha das matrizes $A^{j}$ e $B^{j}$, pretende-se, com o auxílio dos artigos [3,6], criar Spline Wavelets com um formato semelhante ao de um sinal do feto. Assim, ao realizar a decomposição de um ECG, seria possível separar apenas os sinais cardíacos do feto, que é o objeto de análise dessa pesquisa.

Outra possibilidade é a criação de Spline Wavelets com formato semelhante ao sinal cardíaco da mãe. Desse modo, seria possível remover o sinal cardíaco materno e após isso aplicar algum método para remover o ruído, ficando apenas com o sinal cardíaco do feto. Os testes inicialmente serão feitos com sinais disponibilizados no banco de dados da PhysioNet[7].

Palavras-chave: Splines, Wavelets, Análise de sinais

\section{Referências}

[1] Alexandre Bolzan, Giovani Baratto, Alice Kozakevicius, Estimativa do Sinal Fetal FECG do Eletrocardiograma Abdominal Materno Utilizando Wavelets, CNMAC 2010

[2] Alexandre Bolzan, Giovani Baratto, Alice Kozakevicius, Análise de sinais cardíacos Feto-Mãe baseada em Wavelets Packets, CNMAC 2012

[3] Dana Cerná, Václav Finek, Martina Simunková, A Quadratic Spline-Wavelet Basis on the Interval, Programs and Algorithms of Numerical Matematics 16, 29-34, 2013

[4] Jothi S H, Prabha K H. Fetal Electrocardiogram Extraction Using Adaptive Neuro-fuzzy Inference Systems and Undecimated Wavelet Transform. IETE J Res 2012;58:469-75

[5] L. D. Lathauwer, B. D. Moor, and J. Vandewalle, Fetal electrocardiogram extraction by blind source subspace separation, IEEE Med Biol Eng Comput, 47(5), 567-572, 2000

[6] Mohammad Niknazar, S. R. Mousavi, B. Vosoughi Vahdat, Detection of Characteristic Points of ECG using Quadratic Spline Wavelet Transform, 3rd International Conference on Signals, Circuits and Systems, 2009

[7] http://www.physionet.org/physiobank/

[8] Eric J. Stollnitz, Tony D. DeRose, and David H. Salesin. Wavelets for computer graphics: A primer, part 1. IEEE Computer Graphics and Applications, 15(3):76-84, May 1995.

[9] Eric J. Stollnitz, Tony D. DeRose, and David H. Salesin. Wavelets for computer graphics: A primer, part 2. IEEE Computer Graphics and Applications, 15(4):75-85, July 1995. 